\title{
e-Phaïstos
}

e-Phaïstos

Revue d'histoire des techniques / Journal of the history

of technology

VIII-1 | 2020

Écomusée : une expansion internationale

\section{Les Écomusées du patrimoine industriel au Mexique : des institutions encore actuelles?}

De l'influence française à nos jours

Industrial Heritage in Mexico. The Eco-museums revisited

Humberto Morales Moreno

\section{(2) OpenEdition}

Journals

Édition électronique

URL : http://journals.openedition.org/ephaistos/7439

DOI : 10.4000/ephaistos.7439

ISSN : 2552-0741

Éditeur

IHMC - Institut d'histoire moderne et contemporaine (UMR 8066)

Référence électronique

Humberto Morales Moreno, «Les Écomusées du patrimoine industriel au Mexique : des institutions encore actuelles? », e-Phaïstos [En ligne], VIII-1 | 2020, mis en ligne le 29 avril 2020, consulté le 06 mars 2021. URL : http://journals.openedition.org/ephaistos/7439; DOI : https://doi.org/10.4000/ ephaistos.7439

Ce document a été généré automatiquement le 6 mars 2021.

Tous droits réservés 


\section{Les Écomusées du patrimoine industriel au Mexique : des institutions encore actuelles?}

De l'influence française à nos jours

Industrial Heritage in Mexico. The Eco-museums revisited

Humberto Morales Moreno

« Pour Foucault, les hétérotopies par excellence

étaient les espaces appelés disciplinaires, les institutions ayant historiquement pour objet de discipliner les masses (...). Mais ce vocable peut

s'appliquer d'une manière plus générale à la relation entre la forme d'organisation de l'espace et celle de la société et du savoir, ou pour parler

en termes sémiotiques, entre une forme du

signifiant et une forme du signifié1. »

1 Le concept d'écomusée a été introduit en France en 1971 durant la $9^{e}$ conférence de l'ICOM, lorsque le ministre de l'Environnement a repris dans son discours le terme que venait de forger Hugues de Varine-Bohan, avec l'aide de G. H. de Rivière. Il soulignait une nouvelle vague autour du projet original de musée, qui venait d'être inauguré en Bourgogne (Nord-Est de la France). Un groupe de personnes avait travaillé avec des idées radicales et novatrices autour des villes du Creusot et de Montceau-les-Mines, lieux affectés par une crise des mines de charbon et de la sidérurgie. La population locale des villes minières, soutenue par les professionnels des musées, avait récupéré plusieurs espaces industriels abandonnés. L'Écomusée du Creusot-Montceau-les-Mines eut l'idée tout à fait novatrice à l'époque d'utiliser le paysage comme un musée vivant, surtout dans le contexte des pays latins d'Europe. Un peu plus de 45 ans après l'élaboration de ce projet, l'impact de l'Écomusée reste plus proche d'un débat académique en dehors de France plutôt que d'une vraie réussite sur place. En même temps, aux Amériques, ont été consolidées dans les deux dernières décennies du $\mathrm{XX}^{\mathrm{e}}$ 
siècle des adaptations d'écomusées remarquables tels que le Parc National historique de Lowell, aux États-Unis, qui offre toute une muséification complète d'une ville textile bâtie autour du coton².

La première génération des musées en dehors de la tradition objet/visiteur a commencé au Nord de l'Europe, dans les pays scandinaves, avec les musées territoriaux "open air » (Skasen en Suède) et en France, où l'on inaugurait les écomusées dits de deuxième génération, les musées de la récession, de la combinaison de l'industrie, de la culture et de la nature avec des programmes de gestion de territoire comprenant la participation active des habitants en tant que gardiens de leur identité locale. En Allemagne, dans la région de la Ruhr, en lien avec l'environnement et la récupération du patrimoine industriel, on rencontre les mines et usines du Zollverein, Duisbourg et Gelsenkirchen, qui sont des icônes de la conservation du patrimoine industriel dans la transformation du territoire. En Espagne, une fondation très récente (Fondation Atalaya) travaille en faveur de la réhabilitation et de la récupération de la gestion des ressources du patrimoine et de l'exploitation minière. Le parc industriel de Rio Tinto à Huelva est, sans aucun doute, un exemple essentiel de l'histoire de l'exploitation minière, mais également un exemple instructif des nouveaux concepts de la muséification industrielle. Parcs Naturels, Musées et Écomusées ont évité de rester en tant que témoins isolés, qui conserveraient uniquement des cheminées, pour se tourner vers des contenus fonctionnels partiellement considérés, associant le patrimoine industriel à la nature, dans une véritable politique de médiation du patrimoine culturel. La tendance a été évolutive en allant dans le sens de donner plus de valeur à la performance sur place, mais aussi en se tournant vers la notion récente de parcs patrimoniaux. En lien avec la banalisation de la notion de parcs thématiques, nous trouvons d'autres cas de dérives plus prosaïques dans le sens commercial du terme. Cette troisième génération des parcs du patrimoine profite de l'intérêt croissant pour le tourisme industriel et culturel, accompagné de la participation de la population locale. Un parc du patrimoine est une entité complexe qui propose de traverser la volonté de développement économique, les intérêts sociaux et les zones de préservation de l'environnement en nette détérioration. Ces projets sont développés dans des zones, régions ou grands corridors géographiques. Le parc du patrimoine (Schuster, Sabaté, Álvarez) peut être considéré comme un « projet qui tente d'unir les ressources culturelles pour la gestion des territoires à la bonne gestion de ces ressources $^{3} »$.

\title{
L'évolution des écomusées au Mexique
}

\author{
« El Eco-museo entiende que es una provocación a la \\ memoria y el instrumento de una toma de conciencia. \\ Ahí la sociedad (una comunidad) es invitada a tomar \\ conciencia de un patrimonio. El museo, se dice, no \\ tiene visitantes, tiene habitantes. El objetivo: \\ "movilizar el patrimonio para fines creativos y ya no \\ solo museísticos ${ }^{4}$.»
}

3 Cet article aborde la question de l'évolution de quelques écomusées au Mexique qui ont débuté à partir des années 1980, pour présenter les nouvelles propositions dans le cadre des «nouveaux musées» patrimoniaux d'aujourd'hui. Le projet du Centre 
d'Interprétation des espaces liés à la première usine textile d'Amérique Latine à Puebla, en comprenant outre les musées, les analyses en architecture industrielle, histoire des techniques, logements des travailleurs et bâtiments sociaux réutilisés, traditions et coutumes préservées, dépasse la notion de bâtiments isolés et redonne à la notion d'écomusée toute son importance, en essayant de mettre en valeur de véritables paysages industriels en tant que paysages culturels. L'histoire de l'influence française des écomusées industriels au Mexique a commencé à partir du projet de l'Université Autónoma de Puebla, sous la gestion de M. le professeur Samuel Malpica, dans les années 1982-1984, avec la création de l'Écomusée de Metepec, au sud-ouest de l'État de Puebla (plateau central du Mexique). Un véritable projet de mise en valeur d'un village et de sa vie villageoise d'origine ouvrière a vu le jour. À cause de la récession mexicaine (1976-1984), le site se retrouvait dans des conditions précaires après l'échec de l'administration du syndicat ouvrier de l'usine textile, et de sa fermeture définitive en 1967.

Fig.1. Ouvrier de l'usine textile Metepec

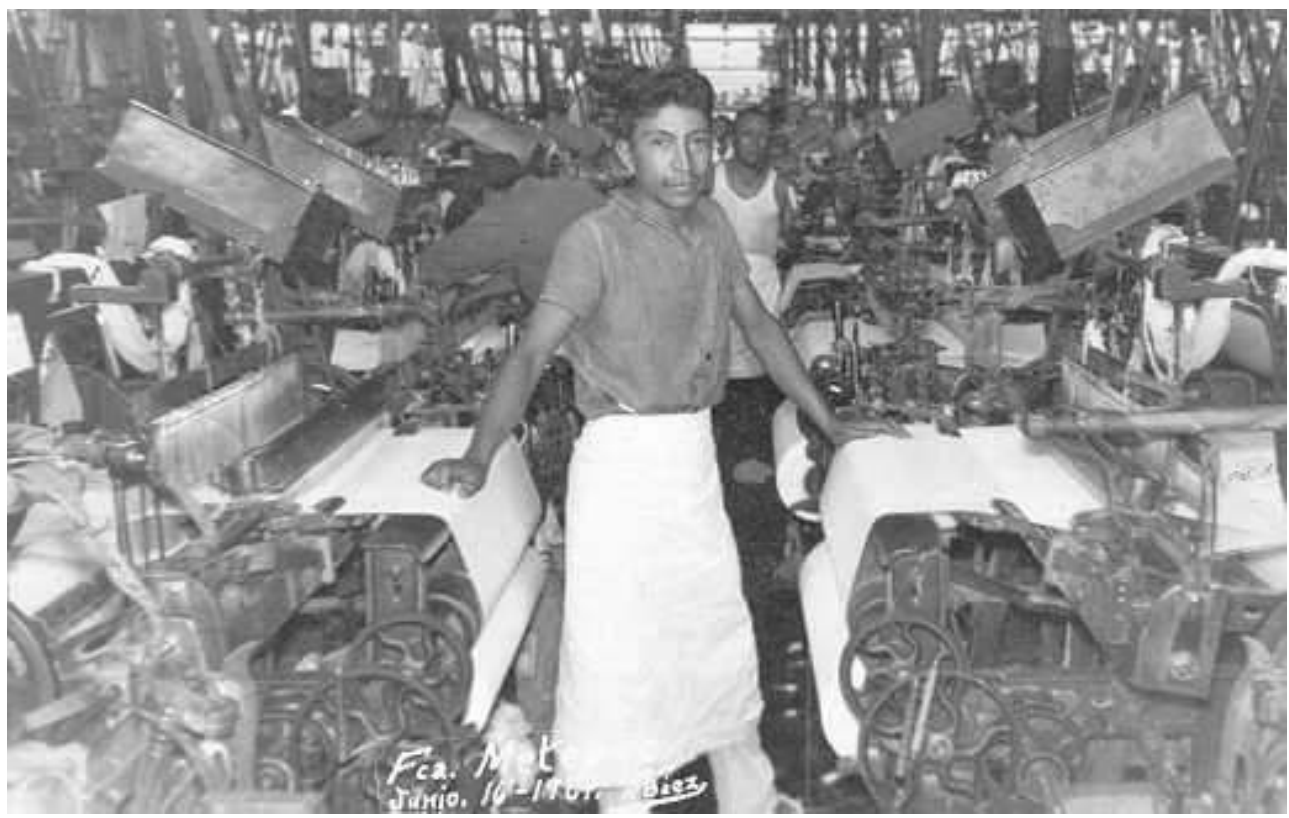

L'usine Metepec se situe au sud-ouest de l'État de Puebla. Date de la photo : c.1907.

Collection particulière de la famille de Rafael et Félix Vede. Tous droits réservés.

4 Metepec était un des plus importants sites industriels textiles de la période $d u$ Porfiriato, (1880-1910), c'est-à-dire de la première vague de l'industrialisation mexicaine. Aujourd'hui, le site fait partie d'un centre de tourisme populaire ouvert au public, avec un spa et des espaces de loisirs familiaux qui entourent la vieille usine et ses bâtiments, les maisons des employés et un petit musée ouvrier où subsiste actuellement le centre de documentation et d'archives historiques de la Compagnie Industrielle de l'Atlixco S. A., société qui a géré l'usine pendant les années 1902-1940 environ, et la communauté ouvrière de Metepec, dès les années 1940 jusqu'à la fermeture en 1967. À vrai dire, Metepec est plutôt un centre culturel touristique avec une ville ouvrière à côté et un petit musée de la vie ouvrière, mais sans aucun centre d'interprétation. De plus, la population du village reste un peu éloignée aux alentours de la municipalité avec très peu de liens par rapport au site patrimonial ${ }^{5}$. 
5 Le deuxième projet est le musée de mines Real del Monte et Pachuca qui gère quatre musées : le musée et les archives historiques de la Compagnie Real del Monte et Pachuca ; le site minier d'Acosta qui reste le premier musée minier avec un projet de centre de documentation; le site La Dificultad qui montre très bien la transition entre la vapeur et l'électricité dans une petite et tardive mine exploitée à partir de 1865 et qui dispose déjà d'un petit centre d'Interprétation ; le Centre Culturel Nicolás Zavala, premier musée de la médecine du travail chez les travailleurs miniers dès 1907. L'ensemble des sites miniers de Real del Monte fait partie de la transition entre l'écomusée de la récession et $\mathrm{du}$ monde du travail et le parc patrimonial moderne. C'est un mélange parfois traditionnel d'objets/visiteurs et un parcours thématique avec la participation du village in situ et un dialogue permanent avec la nature ${ }^{6}$.

Fig.2. La mine d'Acosta

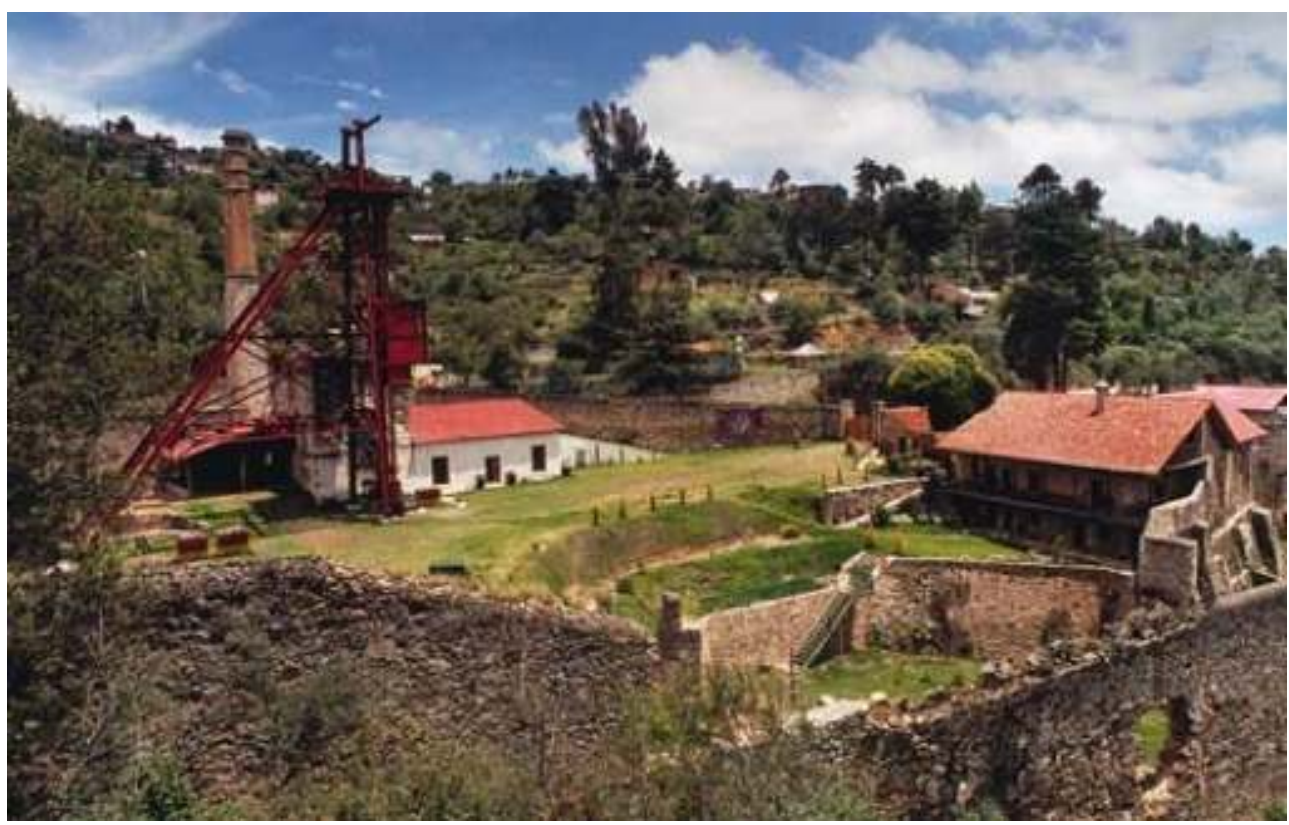

Désormais patrimonialisé, le site fait partie de l'ensemble mis en valeur et géré par le musée des mines Real del Monte et Pachuca.

Tous droits réservés

Le troisième projet est l'ancien musée textile de Bellavista, situé non loin de la ville de Tepic, État de Nayarit, vers la côte ouest du pays. L'usine textile fut déclarée monument historique par l'INAH en 1984 (elle a été fondée en 1841, avec une très forte influence belge dans sa construction) et très peu de temps après, un musée de site ouvrait, avec un petit centre d'archives historiques et une salle de documentation articulée autour de la mémoire intangible du monde du travail (premier syndicat de femmes ouvrières du Mexique et première grève moderne au cours de l'année 1905). Malheureusement, comme beaucoup de projets locaux au Mexique, le manque de soutien et de gestion de ces sites industriels a fini par banaliser les espaces patrimoniaux en faveur du commerce ou de l'aménagement touristique, sans aucune référence muséologique. Aujourd'hui, un gros projet, mené par l'État de Nayarit, entend bâtir un Centre Culturel et des Arts, afin de profiter des installations de l'usine et les préserver en tant que patrimoine culturel, mais apparemment sans aucun lien avec la population et sans y intégrer un travail de création d'un centre de documentation et d'interprétation ${ }^{7}$. 
Fig.3. L'ancienne usine textile Bellavista, déclarée monument historique en 1984

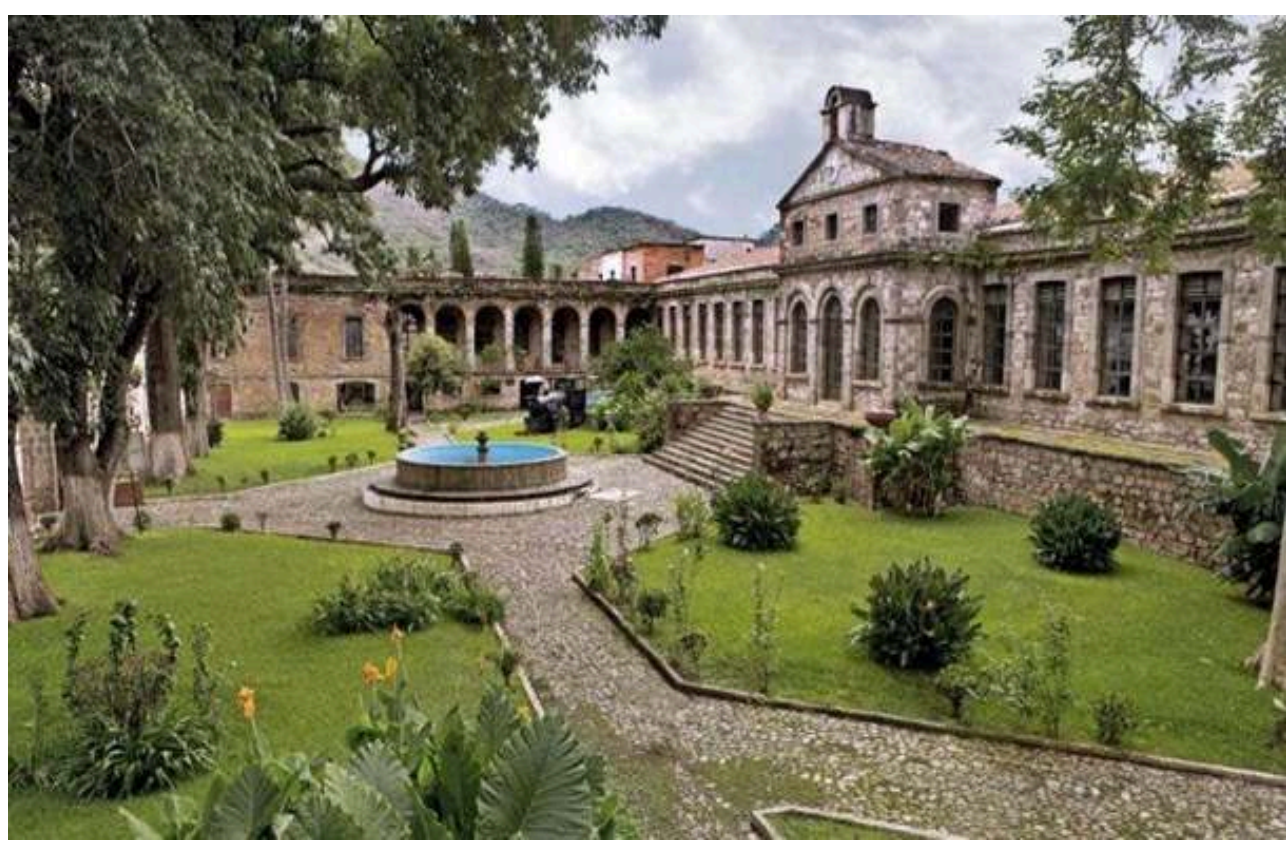

L'usine a été fondée en 1841. Intervention actuelle du Ministère de la Culture du Gouvernement de l'État de Nayarit.

Tous droits réservés.

\title{
Les musées communautaires
}

7 Au Mexique, le musée communautaire est une forme d'écomusée qui a pour tradition une participation importante de la population. L'organisation des musées communautaires du Mexique date de l'année 1994 et le but identitaire est évident:

\begin{abstract}
«El museo comunitario es un instrumento de la acción organizada de comunidades, pueblos originarios y mestizos, que buscan manejar su patrimonio cultural por sí mismos para valorar el pasado y construir su propio futuro. Un museo comunitario es un espacio donde los integrantes de la comunidad construyen un autoconocimiento colectivo, propiciando la reflexión, la crítica y la creatividad. Fortalece la identidad, porque legitima la historia y los valores propios, proyectando la forma de vida de la comunidad hacia adentro y hacia fuera de ella. Fortalece la memoria que alimenta sus aspiraciones de futuro ${ }^{8}$. »
\end{abstract}

Le Mexique est le pays du continent américain qui a le plus gros ensemble de musées communautaires. Il y a au moins deux cents communautés territoriales qui soutiennent ces projets plus proches de l'héritage identitaire que de la muséification patrimoniale. L'anthropologue Raúl Méndez Lugo est le responsable et créateur de cette muséologie tripartite au Nayarit : Écomusée, Territoire et Communauté.

En dehors de cette tendance actuelle concernant la muséification évolutive au Mexique, face à l'influence un peu lointaine déjà du modèle français des écomusées, nous avons plusieurs exemples isolés de sites industriels transformés en espaces patrimoniaux, 
avec une très forte influence des musées traditionnels : objets/visiteurs, sans aucune relation habitant/activité, ou, en tous cas, des interventions du type parc thématique de loisirs touristiques, mais en l'absence de toute sorte d'interprétation sur place. Le cas du parc Fundidora de Monterrey montre très bien cette expérience et les tendances actuelles d'une certaine muséologie industrielle mexicaine.

\title{
Écomusées, parcs patrimoniaux et les nouveaux musées industriels. Le projet de La Constancia Mexicana
}

\author{
« Le musée, en tant qu'institution en état de \\ renouvellement constant, est avant tout un lieu \\ de fermentation, de communication et de \\ diffusion ; il doit avoir pour objectif d'enrichir les \\ perspectives du visiteur et d'établir des liens avec \\ le public et ses exigences ${ }^{9}$."
}

$10 \mathrm{Au}$ cours des dernières décennies, la sémiotique contemporaine et les théories de la communication ont accordé aux études muséales le rôle fondamental de l'observateur et de l'utilisation de l'espace pour associer les pièces ou combler les lacunes entre un fragment et un autre. Avec ces approches, un changement communicatif a eu lieu en muséologie, mettant l'accent sur les qualités d'interprétation de la représentation muséologique. Dans la production de sens intervient toujours la production symbolique. Les objets muséographiques ne sont rien de plus que des représentations de l'histoire, des traces susceptibles d'être vues ou lues de plusieurs façons. L'objet muséographique acquiert un surplus de sens (P.Ricœur) dans le cadre de l'observation.

La réflexion en muséologie de la décennie 1970-1980 a conduit à une critique du musée traditionnel. Mais jusqu'à présent, aucune association internationale des musées n'est revenue sur son approche fondamentale : l'habilitation cognitive de tout observateur indépendamment des procédures de qualités administratives, de gestion et des approches pédagogiques des expositions.

12 En dépit de l'impossibilité de construire des récits linéaires, non fragmentés, les muséographies actuelles au Mexique ont essayé de changer les modes de représentation et, par conséquent, les services au public. Dans beaucoup de ces musées, le point de vue dominant vise différentes approches de la revivification du passé plutôt que des récits d'apprentissage par cœur. Même dans de nombreux musées d'art ou de science, il y a une tendance vers une pleine conscience de transformer les observateurs en peintres quasi-potentiels ou presque scientifiques. La technologie interactive offre la possibilité de mettre à jour l'historiographie. Au lieu de cela, les peintures murales, par exemple, sont devenues elles-mêmes des œuvres d'art, des références fondamentales d'une certaine conscience esthétique du $\mathrm{XX}^{\mathrm{e}}$ siècle. Pour cette raison, il est impossible de les refaire. D'où l'importance de choisir des matériaux et des techniques d'exposition qui permettent la réinterprétation systématique et la maintenance technique optimale à long terme et à faible coût.

13 En outre, depuis des décennies, les «musées en plein air ", "interactifs", "écologiques» et "communautaires» ont remis en cause les musées dans leur fonction rhétorique, dans leur façon de dire des choses, rejetant la rigidité pédagogique 
(silencieuse) du musée moderne traditionnel. Une sorte de transition post-moderne du musée du savant est opérée vers le musée persuasif, du musée des objets / fragments au musée pittoresque.

Quel est le principe de base de tout musée moderne? Son point de départ est la conception d'un programme narratif dont le but est que les sujets (visiteurs, utilisateurs ou observateurs) acquièrent une certaine autonomisation cognitive (une connaissance historique, la connaissance esthétique, les connaissances scientifiques, etc.). La Muséologie est finalement un dispositif de vision, créant un champ de visibilité et, par conséquent, de l'intelligibilité. Ce qui est en jeu est la relation entre doing/seeing et seeing/doing. Le doing/seeing est en lien avec l'organisation de l'espace et de l'exposition elle-même et le seeing/doing est en lien avec le parcours effectué par le visiteur ${ }^{10}$.

En vertu de cette orientation théorique et en ajoutant la contribution que le concept d'écomusée a donné aux projets de récupération et de mise en valeur du patrimoine industriel du Mexique, en tant que patrimoine matériel et immatériel d'une communauté et d'un territoire, une équipe de chercheurs multidisciplinaires a mis au point une proposition de réutilisation du site industriel de la Constancia Mexicana, à Puebla. Celui-ci se présente en tant que lieu de mémoire, espace de la première usine mécanisée de l'Amérique latine fondée en 1835 avec un petit village ouvrier (company town), sous l'influence de projets utopiques catalans, français et belges de la première moitié du XIX ${ }^{e}$ siècle. Tout cela est issu de la vision d'un agriculteur, Don Estevan de Antuñano, avec une forte affiliation pragmatique et utilitaire. Il a proposé une République de l'Industrie dans le style de Saint-Simon, au Mexique ${ }^{11}$. À partir de cette date, le site industriel de La Constancia Mexicana a évolué comme une ville industrielle textile entre 1835-1991. Le Syndicat ouvrier qui l'a administré depuis 1972 a décidé de fermer ses portes, et la propriété connaît alors un processus d'abandon et de détérioration. Après avoir été négligée pendant 20 ans, l'ancienne usine textile a retrouvé sa splendeur historique à travers la mise en œuvre de projets ambitieux, dont le dénominateur commun est la musique, l'art et l'histoire et ceci grâce à un travail coordonné entre les universitaires, la société civile, les entreprises et le gouvernement. 
Fig. 4. Réutilisation des maisons ouvrières de la Constancia Mexicana

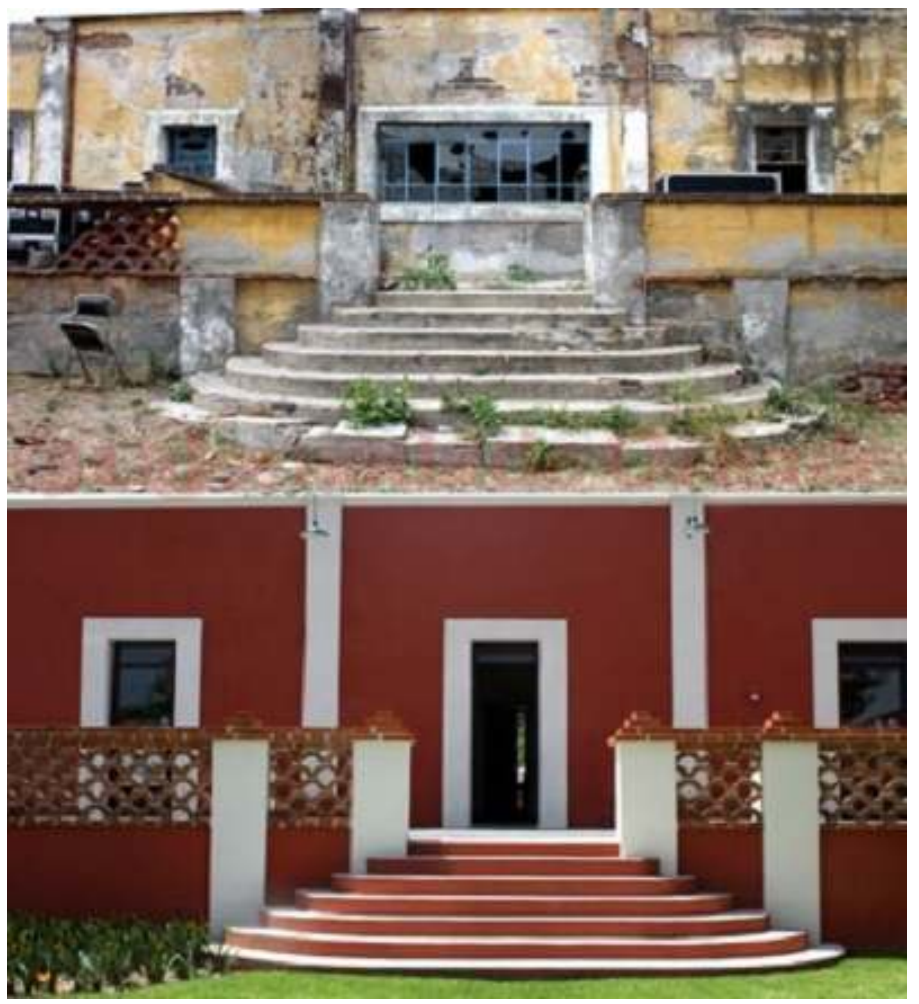

Exemple de la réhabilitation du site, en cours.

Tous droits réservés

\title{
Le Centre d'Interprétation. La nouvelle vague au-delà de l'écomusée
}

\author{
«L'espace du musée semble aussi être \\ nécessairement une traduction de l'espace : \\ fréquemment dans les discussions en cours sur \\ les musées, le manque et l'excès de \\ communication sont tout autant déplorés l'un \\ que l'autre ${ }^{12}$. »
}

En Septembre 2001, le gouvernement de l'État de Puebla a déclaré d'utilité publique le domaine du site industriel, La Constancia Mexicana. Aujourd'hui, 18 ans après cette décision, le bâtiment historique, y compris son environnement immédiat, continue d'être en cours de restauration et de réutilisation. Le patrimoine culturel acquiert sa véritable valeur quand il est utile à la société qui l'a généré, en tant qu'héritage identitaire. C'est pour cela qu'à partir de 2011, des mesures visant à réutiliser l'espace industriel comme un parc du patrimoine ont été prises. La coordination et les efforts menés par les gouvernements fédéral, provinciaux et municipaux en relation avec le secteur privé, à travers le programme Orquestas musicales Esperanza Azteca, ont permis d'offrir aux enfants et aux jeunes la possibilité d'accéder au monde de la musique par l'apprentissage et la maîtrise d'un instrument de musique et la découverte de talents. Ainsi, l'espace où, auparavant, on écoutait la rythmique des métiers industriels a été transformé en un monde musical. La zone de fabrication a été réhabilitée en une zone 
de travail créatif et on a repris les idéaux sociaux du complexe industriel par la création d'un centre de formation pédagogique et artistique : « La nouvelle utopie ».

Les vestiges industriels de l'ancienne usine ont cédé la place à la naissance d'un complexe culturel pionnier au Mexique avec quatre musées. La Maison de la Musique de Vienne à Puebla est le résultat d'un projet global de sauvetage du bâtiment le plus vieux de l'usine. Depuis Janvier 2015, avec la présence de la Maison de la Musique de Vienne, le domaine est un espace unique dans le pays. C'est un choix privilégié en faveur de la connaissance, la jouissance et le loisir de tous les publics. La deuxième vague de musées est constituée par l'ouverture de la Maison des Marionnettes du Mexique et du Musée des Enfants, vers la fin 2015. Depuis 2016, la réutilisation du site s'est achevée avec la création de la Maison de la Musique mexicaine et le lancement d'une opération de sauvetage sans précédent des espaces verts entourant le magnifique centre culturel. Aux Sons, rythmes, jeux, histoire et expositions s'ajoute un aspect supplémentaire : les anciens travailleurs de l'usine sont aussi de retour sur le campus avec la création d'un centre des archives de la parole ouvrière. Dans le cadre d'une approche différente, mais éminemment sociale, par des tournées et avec leur aide, l'origine de la République industrielle et son fondateur sont mis en avant.

Le sauvetage des traces de la production de La Constancia Mexicana a offert une gamme de matériaux qui reflètent la preuve des spécificités historiques utilisées dans le traitement des matières premières par trois éléments de base : le travail humain, des techniques et des machines. En ce sens, outre le processus méticuleux de récupération architecturale, l'inspection archéologique de l'espace a été principalement destinée aux quatre domaines qui, dans leur ensemble, définissent historiquement le site industriel. Il s'agit de veiller à la préservation de ces témoignages dans la perspective d'un parc patrimonial avec la participation de la communauté des anciens travailleurs, dans un dialogue permanent avec la nouvelle proposition où la gestion semble assurée par la dimension culturelle et touristique, sans pour autant banaliser ces quatre axes d'identité : a) Domaines d'activités productives ; b) Zones de loisirs et de vie sociale ; c) Biens meubles; d) Témoignages du monde du travail.

Le processus a connu différentes étapes, de l'identification à l'enregistrement, l'évaluation et la conservation. Elles ont permis la réutilisation et la valorisation des produits industriels historiques. La Constancia Mexicana, à travers la mise en œuvre d'un Centre d'Interprétation qui n'en est qu'à ses débuts, garantira d'aller au-delà de la récupération architecturale nécessaire. Elle assumera l'un des dilemmes les plus délicats présentés par la récupération du patrimoine industriel aujourd'hui : permettre des stratégies pour les relier de façon permanente à la société.

En ce sens, l'usine conçue comme génératrice d'histoire sociale devient un dispositif social au-delà de sa dimension patrimoniale. Elle doit être expliquée, comprise et surtout interprétée. Des Centres d'Interprétation ont été imaginés depuis sa création au milieu du $\mathrm{XX}^{\mathrm{e}}$ siècle, solution favorisant la gestion de la diversité, des ressources culturelles et naturelles.

21 Pour cette raison, l'approche en vue de créer un Centre de Documentation complète cet effort institutionnel. Il permettra de consolider le patrimoine industriel en tant que bien culturel dont la conservation n'est pas considérée comme un chef-d'œuvre. Il devra comprendre l'histoire sociale et économique d'une époque. Pour cette raison, le patrimoine industriel devrait être intelligible aux yeux de la population et il est nécessaire de l'exprimer dans toute sa dimension technique et sociale. 
Fig 5. Le site Constancia Mexicana et les nouveaux musées

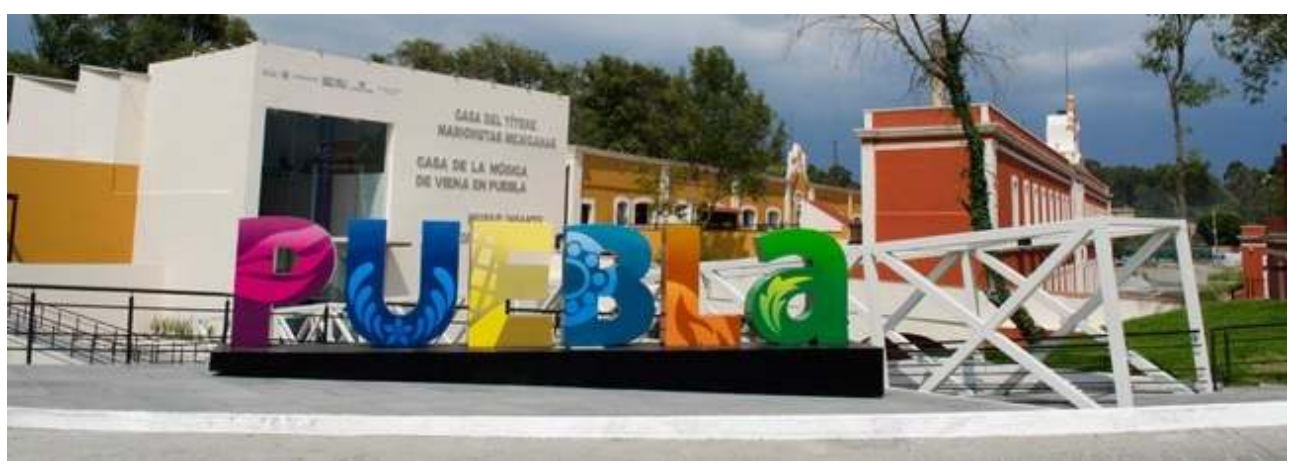

Tous droits réservés proposera une contextualisation des différents stades de l'histoire de la production et du monde du travail : la ferme ; le moulin à blé ; l'usine textile ; le monde du travail.

\section{En conclusion} spatialement distincts, mais inséparables comme preuve du passé : le musée lui-même et les composants auxiliaires de l'ancienne usine, répartis sur cinq hectares. Parallèlement à la visite thématique et chronologique du musée, l'ancienne usine a encore assez d'espaces authentiques qui recréent et comprennent le processus de production et la vie de milliers de travailleurs depuis plus d'un siècle et demi. Beaucoup d'entre eux ont vécu et sont morts dans le complexe productif. Il favorisera les conditions d'apprentissage créatif et contribuera à diffuser au grand public l'importance de l'héritage culturel des biens patrimoniaux existants. Lorsque l'on considère cet ensemble comme un Centre d'Interprétation, les biens et les vestiges industriels acquièrent des significations différentes qu'informent, soulignent et motivent la connaissance et la conservation. Il aura donc pour but de protéger les trois fonctions de base qui tournent autour du paysage et les biens industriels de l'ancienne usine : préservation/réutilisation; divulgation/apprentissage ; recherche/ documentation.

Les visiteurs/acteurs pourront découvrir des informations et des expériences importantes directement liées aux produits industriels de la place. Ses objectifs seront axés sur les significations précédentes in situ et comparés avec l'interprétation. Grâce à des visites des espaces et des vestiges originaux, les différents publics seront informés de l'importance du site, mais à travers l'effort de sensibilisation, ils auront conscience de l'urgence à assurer la protection, la divulgation et l'étude ${ }^{13}$. 


\section{BIBLIOGRAPHIE}

BELLAIGUE Mathilde, «El eco-museo como posible medio de integración. », BONFIL CASTRO Ramón et NESTOR GARCIA Canclini: Memorias del simposio: Patrimonio, Museo y Participación Social. México, INAH, Colección Científica. 1993

BORGES J. Marcelo y SUSANA B. Torres (eds.), Company Towns, USA, Palgrave, Macmillan, 2013

Consejo Estatal para la Cultura y las Artes de Puebla, Dirección de Proyectos Especiales, Archivo fotográfico. Puebla, 2012

FERNANDEZ Miguel Ángel, Historia de los museos de México. México, promotora de comercialización directa, 1988

GAMBOA Leticia, « La Constancia Mexicana. De la fábrica, sus empresarios y sus conflictos hasta los años de la posrevolución », Tzintzun, Revista de Estudios Históricos, Enero- junio 39, 2004, p. 93-112

GOMEZ Jorge, « Arqueología y Museología aplicada al caso de la Constancia Mexicana », Arqueología Industrial, 5, 2002, p.26-29

MATTELART A., Diversidad Cultural y Mundialización. España, Paidós, 2002

MORALES MORENO Humberto, « Proyectos utópicos y el sistema industrial en México en el siglo XIX, La Constancia Mexicana », Arqueología Industrial, número especial 5, 2002, p.12-24

MORALES MORENO Humberto y OSCAR ALEJO García, « El complejo cultural de La Constancia Mexicana », in Luiz Manoel GAZZANEO (ed.), Patrimonio e Paisagem, Brasil, UFRJ, 2012. p.190-210

MORALES MORENO Humberto y SERGIO Niccolai (editores), La Cultura Industrial de México. Memoria del $1^{\circ}$ Encuentro Nacional de Conservación del Patrimonio Industrial, CMCPI. A.C.- Puebla, FFYL-BUAP, 2003

MORALES MORENO Humberto, Oficios urbanos en paisajes agrarios en el altiplano central mexicano en la segunda mitad del siglo XVIII, in Francisco Javier Rodríguez Garza (coord.), Protoindustrialización, industrialización y desindustrialización en la historia de México, México, UAM-A, 2009

MORALES MORENO Humberto, « Desafios de la museología contemporánea: La "desovietización” museográfica de México ", in LACARRIEU Mónica y ALVAREZ Marcelo (Coords.), La (indi) gestión cultural. Una cartografia de los procesos culturales contemporáneos. Buenos Aires, Ediciones Ciccus-La Crujía, 2002

MORALES MORENO Humberto Luis Gerardo, Orígenes de la museología Mexicana, México, Universidad Iberoamericana, 1994

Proyecto de intervención y Reutilización de la Ex Fábrica La Constancia Mexicana, in Consejo Estatal para la Cultura y las Artes. Gobierno del Estado de Puebla, Puebla, Pue, 2009

RICOEUR Paul, Le conflit des interprétations. Essais d'herméneutique I, Paris, Le Seuil, 1969

SANCHEZ FLORES Ramón, « La Constancia Mexicana. Tecnología textil en México. La primera fábrica mecanizada entre 1835-1840», Arqueología Industrial, 5, número especial, 2002, p.5-12

SANCHEZ Andrés, « La Constancia Mexicana y la Arqueología Industrial », Arqueología Industrial, 5, número especial, 2002, p.29-35. 
Términos de referencia para elaborar el proyecto ejecutivo museológico y museográfico del Museo Histórico de la Industria Textil La Constancia Mexicana. Consejo Estatal para la Cultura y Las Artes. Gobierno del Estado de Puebla

\section{ANNEXES}

État actuel (2016) de préservation des espaces d'Interprétation du site La Constancia Mexicana

Les espaces d'Interprétation du site La Constancia Mexicana déjà préservés sont :

1. L'accès au village

2. Le logement des travailleurs

3. La Chapelle (culte actif jusqu'à nos jours)

4. L'école élémentaire « Article 123 »

5. L'espace des gardiens

6. Les tourelles de surveillance

7. Les bains communaux des travailleurs

8. Le stockage / entrepôt de matières premières et des produits finis

9. Les voies ferrées

10. La fonderie

11. La station électrique

12. L'atelier mécanique

13. Le chantier de chargement et de déchargement

14. Le jardin de l'usine

15. Le logement des salariés de l'administration

16. Les vestiges du moulin à blé adapté aux usages industriels

17. Le pont d'accès aux entrepôts et de production

18. Le magasin de pièces

19. Les bureaux administratifs

20. Les maisons des employés

21. La façade d'origine

22. La zone des métiers

23. La zone des trochiles

24. Les turbines

25. Les tunnels

26. La zone des balançoires 
27. La zone de préparation de la filature

28. La zone de nettoyage à sec

29. La zone de la chaudière

30. La cheminée

31. La zone d'encollage

32. La menuiserie

34. Le canal et le système de contrôle de la force hydraulique

\section{NOTES}

1. ECO Umberto, «Le musée du troisième millénaire », in ECO Humberto et PEZZINI Isabella, Le musée, demain. Espagne, Casimiro, 2015, p.50.

2. Voir l'hommage à Georges Henri Rivière : Museum, Unesco, Paris, 148, 1985 (Images des écomusées).

3. ÁLVAREZ ARECES Miguel Ángel, "Nuevas miradas al paisaje y al territorio ", Ábaco, 34, segunda época, Gijón, CICEES, 2002, p.17-40 ; SABATÉ Joaquín et SCHUSTER Mark (coords.), Projectant l'eix del Llobregat. Paitsage cultural i desenvolupament regional, Barcelona, Universidad Politécnica de Cataluña, Massachussets Institute of Technology, 2001.

4. QUERRIEN, Les monuments historiques demain, Paris, Direction du Patrimoine, 1987, p.265, Apud : HARTOG François, Regímenes de Historicidad, Presentismo y Experiencias del Tiempo, México, UIA, 2007, p.221.

5. Museo Industrial de Metepec. Benemérita Universidad Autónoma de Puebla. Chargé de projet: Prof. Mariano Castellanos.

6. Archivo Histórico y Museo de Minería. Real del Monte y Pachuca. Chargée de projet: Prof. Belem Oviedo Gámez.

7. Le créateur du premier musée du site textile fut l'Anthropologue Raúl Méndez Lugo, ancien directeur de l'INAH à Nayarit.

8. https://zita-possamai.blogspot.com/2009/12/rede-de-museus-comunitarios-da-america.html (12.06.2018).

9. MONTANER J. M., « Musei del XXI secolo. Nuove tipologie, nuovi rapporti con i visitatori », AA. VV., Lotus 134, Performing Museums, Lotus-Skira, Milano. 2008, Apud PEZZINI Isabella, Sémiotique du nouveau musée, op. cit., p.60.

10. Il faut voir : Proyecto de curaduría y museografía de la ex fábrica textil "La Constancia Mexicana" para la empresa Gardeco/fundación azteca/gobierno del estado de Puebla. enero-mayo de 2012. Il faut remercier M. Luis Gerardo Morales et M. Oscar Alejo pour leur participation dans ce projet.

11. MORALES MORENO Humberto, «Estevan de Antuñano y la República de la Industria. 1835-1910 ", in FOWLER William et MORALES Humberto (eds.), El Conservadurismo mexicano en el siglo XIX, México, BUAP/Saint Andrews University, p.265-303.

12. PEZZINI Isabella, op.cit., p.73-74.

13. MORALES MORENO Humberto et SERGIO Niccolai (eds.). La Cultura Industrial de México. Memoria del $1^{\circ}$ Encuentro Nacional de Conservación del Patrimonio Industrial, CMCPI. A.C. Puebla, FFYLBUAP, 2003. 


\section{RÉSUMÉS}

Dans cette communication, on prétend retracer le parcours de l'évolution de quelques écomusées au Mexique qui ont démarré à partir des années 1980, et présenter les nouvelles propositions dans le cadre des "nouveaux musées " patrimoniaux d'aujourd'hui. Un parc du patrimoine est une entité complexe qui propose de traverser la volonté de développement économique, les intérêts sociaux et les zones de préservation de l'environnement en nette détérioration. Ces projets sont développés dans des zones, régions ou grands corridors géographiques. Le parc du patrimoine (Schuster, Sabaté, Álvarez) peut être considéré comme un « projet qui tente d'unir les ressources culturelles pour la gestion des territoires à la bonne gestion de ces ressources ». A Puebla, le projet de centre d'interprétation de la première usine textile d'Amérique Latine dépasse la notion de bâtiment isolé, en intégrant la totalité de l'espace productif et culturel, musées, architecture industrielle, histoire des techniques, logement des travailleurs, bâtiments sociaux actuellement réutilisés, traditions et coutumes toujours vivantes. Cela relance l'importance des écomusées en essayant de mettre en valeur de véritables paysages industriels en tant que paysages culturels.

This essay shows a brief evolution of some eco-museums in Mexico that started from the 80's of the last century and highlights the new proposals in the mainstream of "new heritage landscapes of today". Heritage Parks is a complex entity that proposes to cross the task of economic development, social interests and environmental conservation areas in clear deterioration. These projects are developed in areas, regions or large geographic corridors. The Heritage Park (Schuster, Sabaté, Álvarez) can be considered a "project that tries to unite the cultural resources for better landscapes management and the proper management of these resources". In Puebla, the project of an interpretation centre of the first latin american textile plant, goes beyond the notion of isolated building and includes museums, industrial architecture, history of technology, housing for workers and social buildings, traditional uses. This revitalizes the importance of ecomuseums in safeguarding old industrial territories as cultural landscapes.

\section{INDEX}

Mots-clés : histoire des techniques, écomusée, patrimoine industriel, patrimoine technique, centre d'interprétation, paysage culturel, patrimoine immatériel

Keywords : history of technology, eco-museum, industrial heritage, industrial landscape, interpretation center, heritage park, intangible heritage

\section{AUTEUR}

\section{HUMBERTO MORALES MORENO}

Directeur du Centre de Recherches d'Histoire Économique et Sociale de l'Institut de Sciences du Gouvernement et Développement Stratégique (ICGDE) de l'Université Autonome de Puebla, Mexique. Les axes de recherche sur le patrimoine industriel sont : Archéologie Industrielle du Mexique ; Histoire économique des entreprises manufacturières ; Écomusées et parc patrimoniaux industriels ; Histoire économique et sociale de l'industrialisation aux Amériques. Publication récente : Necaxa, Pueblo de Luz, Mexique, CFE/Puebla, 2018. (Le Patrimoine Industriel de la première usine hydroélectrique de l'Amérique Latine). 